\title{
Numerical analysis of LEC growth of GaAs with an axial magnetic field
}

\author{
Mingwei $\mathrm{Li}^{\mathrm{a}, *}$, Wenrui Hu ${ }^{\mathrm{b}}$, Nuohu Chen ${ }^{\mathrm{c}}$, Danling Zeng ${ }^{\mathrm{a}}$, Zemei Tang ${ }^{\mathrm{b}}$ \\ a Institute of Power Engineering, Chongqing University, Chongqing 400044, China \\ ${ }^{\mathrm{b}}$ Institute of Mechanics, CAS, Beijing 100083, China \\ ${ }^{\mathrm{c}}$ Institute of Semiconductors, CAS, Beijing 100083, China
}

Received 28 September 2001

\begin{abstract}
A set of numerical analyses for momentum and heat transfer for a 3 in. $(0.075 \mathrm{~m})$ diameter Liquid Encapsulant Czochralski (LEC) growth of single-crystal GaAs with or without an axial magnetic field was carried out using the finite-element method. The analyses assume a pseudosteady axisymmetric state with laminar flows. Convective and conductive heat transfers, radiative heat transfer between diffuse surfaces and the Navier-Stokes equations for both melt and encapsulant and electric current stream function equations for melt and crystal are considered together and solved simultaneously. The effect of the thickness of encapsulant, the imposed magnetic field strength as well as the rotation rate of crystal and crucible on the flow and heat transfer were investigated. (c) 2002 Published by Elsevier Science Ltd.
\end{abstract}

Keywords: Liquid Encapsulant Czochralski (LEC); Growth of GaAs; Magnetic field; Finite-element method; Boron oxide

\section{Introduction}

Semiconductor Gallium arsenide is usually grown in Liquid Encapsulated Czochralski (LEC) puller, in which a cylindrical crystal is pulled from the surface of melt. In order to grow high quality GaAs crystal, an adequate thermal circumstance is very important, which is related to furnace structure and the operating conditions, such as the thickness of encapsultant, the crystal and crucible rotations, as well as the imposed magnetic field. In a typical LEC furnace, the fluid flow, heat and mass transfer are quite complex, i.e. highly nonlinear and strongly coupled with each other. For example, a gas guide (heat shield) changes the path of the gas flow near the melt/gas interface, affects the evaporation rate of oxygen, reduces the radiative heat loss and in turn the

\footnotetext{
${ }^{*}$ Corresponding author. Tel.: +86-023-654-15008; fax: +86023-651-02471.

E-mail address: mwlizao@yahoo.com (M. Li).
}

heater power, temperature distributions and melt flow are also modified [1].

To understand the characteristics of the momentum and heat transfer in an LEC growth of GaAs, some numerical simulations have been performed by neglecting the melt flow [2-5]. Dupret and his coworkers [2,3] predicted the thermal field and the location of the melt/ crystal interface by a conduction-dominated model, to show the dependence of some important output parameters such as the deflection of the interface, the maximum value of Mises invariant and the power input upon the degree of opacity of the oxide layer, i.e. the radiative properties of the encapsulant. Thomas et al. [4] extended the dynamic thermal-capillary model to simulate a low-pressure LEC system for the growth of GaAs, and the predicted crystal shape is in semiquantitative agreement with the experiment. The influence of the Argon gas pressure on the heater power, convex curvature of the growth interface and the maximum thermal stress was studied by both laminar and turbulence models for the gas flow [5]. Actually, the melt flow influences the crystal quality, the crystal and crucible 
rotations, as well as the thickness of the encapsulant, are well known to have an impact on the melt flow and melt/ crystal interface shape [6-9]. Numerical simulation of the flow and heat transfer in the melt and encapsulant and heat transfer in the crystal for LEC growth of GaAs with a steady, uniform, axial magnetic field has been done by Sabhapathy and Salcubean [10] for a $0.15 \mathrm{~m}$ (6 in.) diameter crucible, $0.075 \mathrm{~m}$ diameter crystal, $0.05 \mathrm{~m}$ height melt and $0.02 \mathrm{~m}$ thick encapsulant, with constant crucible sidewall temperature imposed, the melt/crystal interface and melt/encapsulant interface being assumed to be flat and undeformable. Their numerical results showed that the flow in the bulk of the melt is suppressed by the magnetic field, however the convection near the crucible side wall is significant even for a large magnetic field of $0.75 \mathrm{~T}$. Two immiscible horizontal liquid layers: $\mathrm{B}_{2} \mathrm{O}_{3}$ (encapsulant) and $\mathrm{GaAs}$ (melt) are also chosen to study numerically the flow characteristics of thermocapillary convection, in which the buoyancy convection is not taken into account [11]. Meanwhile, Jing and his coworkers [12] investigated the thermocapillary and buoyancy flow in co-axial liquid bridges with interfacial deformation owing to the practical application of the LEFZ method for the growth of GaAs.

In the present paper, a set of numerical simulations on momentum and heat transfer for a $0.075 \mathrm{~m}$ diameter LEC growth of single-crystal GaAs was performed. The effect of the thickness of encapsulant, the imposed axial magnetic field and the rotation rate of crystal and crucible on the flow and temperature field and interface shape were discussed in detail.

\section{Model formulation}

\subsection{Physical model and basic assumptions}

Schematic diagram adopted in the present analyses is shown in Fig. 1, in which the GaAs melt/encapsulant interface shape is calculated by solving the YoungLaplace equation. The following assumptions were introduced:

1. The system is in a pseudosteady state and is axisymmetric.

2. The flow is laminar and both the GaAs melt and the boron oxide encapsulant are incompressible.

3. Thermophysical properties of the melt are assumed to be constant except for the temperature dependencies of surface tension and the dynamic viscosity of boron oxide, $\mu$.

4. No subcooling occurs at the melt/crystal interface and the interface shape coincides with the melting temperature isotherm.

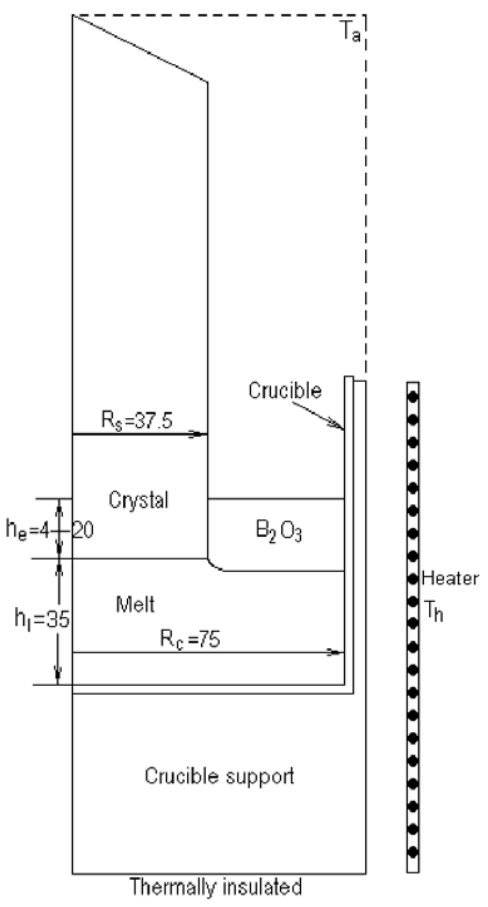

Fig. 1. Schematic model for crucible, crucible support, melt, encapsulant and crystal as used to model an LEC system.

5. The magnetic Reynolds number is so small that the effect of the induced magnetic field on the applied field can be neglected.

\subsection{Mathematical model}

\subsubsection{Governing equations}

With the above assumptions, the governing equations for flow and temperature field are given as follows.

In the melt and encapsulant:

$\nabla \cdot \mathbf{v}_{i}=0$,

$\rho_{i} \mathbf{v}_{i} \cdot \nabla \mathbf{v}_{i}=-\nabla p_{i}-\nabla \cdot \tau_{i}+\rho_{i} g \beta\left(T_{i}-T_{\mathrm{m}}\right) \mathbf{e}_{z}+(J \times B)$,

$\rho_{i} c_{\mathrm{p} i} \mathbf{v}_{i} \cdot \nabla T_{i}=\lambda_{i} \nabla \cdot \nabla T_{i}$.

In the crystal:

$\rho_{\mathrm{s}} c_{\mathrm{ps}} V_{\mathrm{s}} \mathbf{e}_{z} \cdot \nabla T_{\mathrm{s}}=\lambda_{\mathrm{s}} \nabla \cdot\left(\nabla T_{\mathrm{s}}\right)$.

In the other solid materials:

$\nabla \cdot\left(\nabla T_{j}\right)=0$.

Here $T, \mathbf{v}, p$, and $\tau$ are the temperature, velocity vector, pressure and stress tensor, respectively. $T_{\mathrm{m}}$ is the melting temperature, $\rho$ the density, $\lambda$ is the thermal conductivity, $\beta$ the thermal expansion coefficient, $g$ is the gravitational 
acceleration constant, $c_{\mathrm{p}}$ is the heat capacity, $V_{\mathrm{s}}$ is the crystal pulling rate; subscripts $i$, s, and $j$ indicate GaAs melt $(i=1), \mathrm{B}_{2} \mathrm{O}_{3}$ encapsulant $(i=\mathrm{e})$, crystal and other materials, respectively. Vector $\mathbf{e}_{z}$ is a unit vector parallel to the $z$ axis. Product $(J \times B)$ represents the Lorentz force due to the imposed magnetic field, in which $J$ is the electric current density and $B$ is the strength of magnetic field. The electric current density, $J$, is calculated by introducing the electric current stream function $\psi_{j}$ which is determined as

$$
\begin{aligned}
& \frac{\partial}{\partial z}\left(\frac{\xi_{\ell}}{\xi} \frac{1}{r} \frac{\partial \psi_{j}}{\partial z}\right)+\frac{\partial}{\partial r}\left(\frac{\xi_{\ell}}{\xi} \frac{1}{r} \frac{\partial \psi_{j}}{\partial r}\right) \\
& \quad=B_{z} \frac{\partial w}{\partial z}+B_{r} r \frac{\partial}{\partial z}\left(\frac{w}{r}\right)
\end{aligned}
$$

where $\psi_{j}$ is the electric current stream function defined by

$J_{z}=-\frac{\xi_{\ell} B_{0}}{r} \frac{\partial \psi_{j}}{\partial r}$

$J_{r}=-\frac{\xi_{\ell} B_{0}}{r} \frac{\partial \psi_{j}}{\partial z}$

where $\xi$ is the electrical conductivity and $B_{0}$ is the magnetic field strength. The above equations are valid both in the melt $\left(\xi=\xi_{\ell}\right)$ and in the crystal $\left(\xi=\xi_{\mathrm{s}}\right)$.

\subsubsection{Boundary conditions for the temperature field}

At the melt/encapsulant interface:

$-\lambda_{\ell} \mathbf{n} \cdot \nabla T_{\ell}=-\lambda_{\mathrm{e}} \mathbf{n} \cdot \nabla T_{\mathrm{e}}+q_{\mathrm{rad}, \ell}$.

At the encapsulant free surface:

$-\lambda_{\mathrm{e}} \mathbf{n} \cdot \nabla T_{\mathrm{e}}=q_{\mathrm{rad}, \mathrm{e}}$.

At the melt/crystal interface:

$\lambda_{\ell} \mathbf{n} \cdot \nabla T_{\ell}-\lambda_{\mathrm{s}} \mathbf{n} \cdot \nabla T_{\mathrm{s}}=\rho_{\mathrm{s}} V_{\mathrm{s}} \Delta H_{\mathrm{s}} \mathbf{n} \cdot \mathbf{e}_{z}$,

$T_{\ell}=T_{\mathrm{s}}=T_{\mathrm{m}}$.

At the encapsulated crystal side:

$-\lambda_{\mathrm{s}} \mathbf{n} \cdot \nabla T_{\mathrm{s}}=-\lambda_{\mathrm{e}} \mathbf{n} \cdot \nabla T_{\mathrm{e}}+q_{\mathrm{rad}, \mathrm{s}}$.

At the encapsulated crucible side:

$-\lambda_{j} \mathbf{n} \cdot \nabla T_{j}=-\lambda_{\mathrm{e}} \mathbf{n} \cdot \nabla T_{\mathrm{e}}+q_{\mathrm{rad}, j}$.

At the exposed crystal side and top:

$-\lambda_{\mathrm{s}} \mathbf{n} \cdot \nabla T_{\mathrm{s}}=q_{\mathrm{rad}, \mathrm{s}}$.

At the other exposed surfaces:

$-\lambda_{j} \mathbf{n} \cdot \nabla T_{j}=q_{\mathrm{rad}, j}$

In these equations, $q_{\mathrm{rad}, i}$ is the net radiative heat flux per unit area on the surfaces, and is given by

$$
q_{\mathrm{rad}, i}=\varepsilon_{i} \sigma_{B}\left[T_{i}^{4}-\left(\sum_{j} A_{j} \varepsilon_{j} T_{j}^{4} G_{j i}\right) / A_{i} / \varepsilon_{i}\right],
$$

where $G_{j i}$ is Gebhart's absorption factor, which is the fraction of emission from surface $A_{j}$ to $A_{i}$ and absorbed, $G_{j i}$ as well as the view factors between each surface element in the furnace are calculated using our code and $\Delta H_{\mathrm{s}}$ is the latent heat of fusion, $\mathbf{n}$ is the unit normal vector of the interface element and $\epsilon$ is the emissivity.

\subsubsection{Boundary conditions for the flow field and electric} current stream function

At the side wall and the bottom of the crucible:

$u_{\ell}=v_{\ell}=0, \quad w_{\ell}=r \omega_{\mathrm{c}}, \quad \psi_{j}=0$.

At the encapsulated side wall of the crucible:

$u_{\mathrm{e}}=v_{\mathrm{e}}=0, \quad w_{\mathrm{e}}=r \omega_{\mathrm{c}}$.

At the melt/encapsulant interface:

$\mathbf{n} \cdot \mathbf{v}_{\ell}=0, \quad \mathbf{n} \cdot \mathbf{v}_{\mathrm{e}}=0, \quad \psi_{j}=0$,

$\tau_{\ell}: n t-\tau_{\mathrm{e}}: n t=\gamma_{\mathrm{T}} \nabla T_{\ell} \cdot t$,

$\mathbf{t} \cdot \mathbf{v}_{\ell}-\mathbf{t} \cdot \mathbf{v}_{\mathrm{e}}=0$

$\tau_{\ell}: n \mathbf{e}_{\theta}-\tau_{\mathrm{e}}: n \mathbf{e}_{\theta}=\gamma_{\mathrm{T}} \nabla T_{\ell} \cdot \mathbf{e}_{\theta}$,

$\mathbf{e}_{\theta} \cdot \mathbf{v}_{\ell}-\mathbf{e}_{\theta} \cdot \mathbf{v}_{\mathrm{e}}=0$.

At the encapsulant surface:

$\mathbf{n} \cdot \mathbf{v}_{\mathrm{e}}=0$,

$\tau_{\mathrm{e}}: n t=\gamma_{\mathrm{T}} \nabla T_{\mathrm{e}} \cdot t$,

$\tau_{\mathrm{e}}: n \mathbf{e}_{\theta}=\gamma_{\mathrm{T}} \nabla T_{\mathrm{e}} \cdot \mathbf{e}_{\theta}$.

At the melt/crystal interface:

$u_{\ell}=v_{\ell}=0, \quad w_{\ell}=r \omega_{\mathbf{s}}$.

At the encapsulated surface of crystal:

$u_{\mathrm{e}}=v_{\mathrm{e}}=0, \quad w_{\mathrm{e}}=r \omega_{\mathrm{s}}, \quad \psi_{j}=0$.

At the other crystal surface:

$\psi_{j}=0$.

At the axis:

$\psi_{j}=0$.

Here $u, v$, and $w$ are the radial, axial and the azimuthal components of velocity, $\omega_{\mathrm{s}}$ and $\omega_{\mathrm{c}}$ are the angular rotation rates of the crystal and crucible, respectively. Symbols $\mathbf{t}$ and $\mathbf{e}_{\theta}$ are the unit tangential and azimuthal vectors of each interface element, respectively. The melt/crystal interface shape is determined so that Eq. (9d) is satisfied, i.e. the interface coincides with the melting-point isotherm.

\subsection{Numerical procedure}

We define the characteristic scales of the system by $T_{\mathrm{m}}$ and $v_{\ell} / r_{\mathrm{c}}$ for temperature and velocity, $\left(p-p_{0}+\rho_{\ell} g z\right)$ 
for pressure. Then, the system is characterized by the Grashof number $G r_{\ell}=r_{\mathrm{c}}^{3} \beta_{\ell} T_{\mathrm{m}} g / v_{\ell}^{2}$ and $G r_{\mathrm{e}}=r_{\mathrm{c}}^{3} \beta_{\mathrm{e}} T_{\mathrm{m}} g /$ $v_{\ell}^{2}$, Reynolds number $R e_{\mathrm{s}}=2 \pi r_{\mathrm{s}}^{2} \omega_{\mathrm{s}} / v_{\ell}$ and $R e_{\mathrm{c}}=2 \pi r_{\mathrm{c}}^{2} \times$ $\omega_{\mathrm{c}} / v_{\ell}$, Marangoni number $M a_{1}=\gamma_{1} r_{\mathrm{c}} T_{\mathrm{m}} / \mu_{\ell} v_{\ell}$ and $M a_{2}=$ $\gamma_{2} r_{\mathrm{c}} T_{\mathrm{m}} / \mu_{\ell} v_{\ell}$, Peclet number $\left.P e=\rho_{\mathrm{s}} c_{\mathrm{ps}} V_{\mathrm{s}} r_{\mathrm{c}} / \lambda_{\ell}\right)$, Prandtl number $P r_{\ell}=\mu_{\ell} c_{\mathrm{p} \ell} / \lambda_{\ell}$ and $P r_{\mathrm{e}}=\mu_{\mathrm{e}} c_{\mathrm{pe}} / \lambda_{\mathrm{e}}$. These dimensionless numbers are listed in Table 1. By applying the Galerkin finite-element method, this system gives a set of algebraic equations.

In the present work, the diameter and length of the crystal and the heater temperature were given. The crystal pull rate was determined as one of the unknown variables, together with velocities, pressures, temperatures, electric current stream function and the melt/ crystal interface coordinates, by simultaneously solving the set of nonlinear algebraic equations using the Newton-Raphson method. The radiative heat exchange in the semitransparent boric oxide layer is calculated by using the band-energy method [3]. The total number of elements, nodal points and unknowns is 9127,9343 and 40829 , respectively. The sizes of the minimum elements in the melt are $\Delta r_{\min }=0.1774 \mathrm{~mm}, \Delta z_{\min }=0.68 \mathrm{~mm}$. Typical conditions are listed in Table 1, together with the thermophysical properties used in this work.

\section{Results}

To make the comparison valid, the pull rate is rigorously maintained at around $5 \mathrm{~mm} / \mathrm{s}$ for all the results by adjusting the heater temperature properly. The maximum relative error of the pulling rate is less than $1 \%$.

\subsection{Encapsulant thickness}

(1) Marangoni-driven flow. Without the magnetic field and crystal and crucible rotations, the effect of the thickness of encapsulant, $h_{\mathrm{e}}$, on the flow and heat transfer in the case of pure Marangoni-driven flow (hereafter we denote it as Ma-driven flow) is shown in Figs. 2-4. The flow is characterized by a single cell flow in both melt and encapsulant. The change of the thickness does not change the flow pattern (see Fig. 2), but changes the maximum velocity $V_{\max }$ and the average velocity $V_{\text {av }}$ in the melt (see Fig. 3), here $V_{\text {av }}$ is defined as follows:

$V_{\mathrm{av}}=\sum\left(\sqrt{u_{i}^{2}+v_{i}^{2}}\right) / N$,

where $N$ represents the total number of nodes in the melt. In this case the flow is very weak so that the heat transfer in the melt is controlled by thermal conduction. With increasing $h_{\mathrm{e}}$, the total heat input from the crucible side wall, $Q_{\mathrm{c}}$, decreases from 1513 to $1423 \mathrm{~W}$, and the
Table 1

Physical properties and processing parameters

\begin{tabular}{|c|c|}
\hline \multicolumn{2}{|l|}{ Melt } \\
\hline Freezing temperature, $T_{\mathrm{m}}$ & $1511 \mathrm{~K}$ \\
\hline Density, $\rho_{\ell}$ & $5.72 \times 10^{3} \mathrm{~kg} / \mathrm{m}^{3}$ \\
\hline Dynamic viscosity, $\mu_{\ell}$ & $2.79 \times 10^{-3} \mathrm{~kg} / \mathrm{m} \mathrm{s}$ \\
\hline Thermal conductivity, $\lambda_{\ell}$ & $1.78 \times 10^{1} \mathrm{~W} / \mathrm{m} \mathrm{K}$ \\
\hline Specific heat, $C_{\mathrm{p} \ell}$ & $4.34 \times 10^{2} \mathrm{~J} / \mathrm{kg} \mathrm{K}$ \\
\hline Thermal expansion coefficient, $\beta_{\ell}$ & $1.87 \times 10^{-4} 1 / \mathrm{K}$ \\
\hline Latent heat, $\Delta H_{\mathrm{m}}$ & $6.685 \times 10^{2} \mathrm{~kJ} / \mathrm{kg}$ \\
\hline Emissivity, $\varepsilon$ & 0.3 \\
\hline Electrical conductivity, $\xi_{\ell}$ & $8.0 \times 10^{5} \mathrm{~S} / \mathrm{m}$ \\
\hline $\begin{array}{l}\text { Surface tension (under } \mathrm{B}_{2} \mathrm{O}_{3} \text { ) } \\
\text { temperature coefficient, } \gamma_{1}\end{array}$ & $\begin{array}{l}-1.2 \times 10^{-3} \mathrm{~N} / \mathrm{m} \mathrm{K} \\
{[12]}\end{array}$ \\
\hline Prandtl number, $P r$ & 0.068 \\
\hline \multicolumn{2}{|l|}{ Crystal } \\
\hline Density, $\rho_{\mathrm{s}}$ & $5.2 \times 10^{3} \mathrm{~kg} / \mathrm{m}^{3}$ \\
\hline Thermal conductivity, $\lambda_{\mathrm{s}}$ & $7.0 \mathrm{~W} / \mathrm{m} \mathrm{K}$ \\
\hline Specific heat, $C_{\mathrm{ps}}$ & $4.24 \times 10^{2} \mathrm{~J} / \mathrm{kg} \mathrm{K}$ \\
\hline Emissivity, $\varepsilon$ & 0.55 \\
\hline Electrical conductivity, $\xi_{\mathrm{s}}$ & $3.0 \times 10^{4} \mathrm{~S} / \mathrm{m}$ \\
\hline \multicolumn{2}{|l|}{ Boron oxide } \\
\hline Density, $\rho_{\mathrm{e}}$ & $1.648 \times 10^{3} \mathrm{~kg} / \mathrm{m}^{3}$ \\
\hline Dynamic viscosity, $\mu_{\mathrm{e}}$ & $10^{-1.862+3650 / T} \mathrm{~kg} / \mathrm{m} \mathrm{s}$ \\
\hline Thermal conductivity, $\lambda_{\mathrm{e}}$ & $2.0 \mathrm{~W} / \mathrm{m} \mathrm{K}$ \\
\hline Specific heat, $C_{\mathrm{pe}}$ & $1.83 \times 10^{3} \mathrm{~J} / \mathrm{kg} \mathrm{K}$ \\
\hline Thermal expansion coefficient, $\beta_{\mathrm{e}}$ & $9.0 \times 10^{-5} 1 / \mathrm{K}$ \\
\hline Emissivity, $\varepsilon$ & 0.65 \\
\hline $\begin{array}{l}\text { Surface tension temperature } \\
\text { coefficient, } \gamma_{2}\end{array}$ & $\begin{array}{l}3.57 \times 10^{-5} \mathrm{~N} / \mathrm{m} \mathrm{K} \\
{[13]}\end{array}$ \\
\hline Prandtl number, $P r$ & 3568.5 \\
\hline \multicolumn{2}{|l|}{ Graphite crucible } \\
\hline Thermal conductivity, $\lambda$ & $4.2 \times 10^{1} \mathrm{~W} / \mathrm{m} \mathrm{K}$ \\
\hline Emissivity, $\varepsilon$ & 0.9 \\
\hline \multicolumn{2}{|l|}{ Quartz crucible } \\
\hline Thermal conductivity, $\lambda$ & $2.98 \mathrm{~W} / \mathrm{m} \mathrm{K}$ \\
\hline Emissivity, $\varepsilon$ & 0.5 \\
\hline \multicolumn{2}{|l|}{ Other parameters } \\
\hline $\begin{array}{l}\text { Contact angle between melt and } \\
\text { crystal, } \theta_{\mathrm{c}}\end{array}$ & $15^{\circ}$ \\
\hline Rotation rates of crystal, $\omega_{\mathrm{s}}$ & $2-20 \mathrm{rpm}$ \\
\hline Rotation rates of crucible, $\omega_{\mathrm{s}}$ & $12-20 \mathrm{rpm}$ \\
\hline Axial magnetic field, $B$ & $0.0-0.35 \mathrm{~T}$ \\
\hline Ambient temperature, $T_{\mathrm{a}}$ & $1254.0 \mathrm{~K}$ \\
\hline Grashof number, $G r_{\ell}$ & $4.915 \times 10^{9}$ \\
\hline Grashof number, $G r_{\mathrm{e}}$ & $2.366 \times 10^{9}$ \\
\hline Reynolds number, $R e_{\mathrm{s}}$ & $1.812 \times 10^{4} \times \omega_{\mathrm{s}}$ \\
\hline Reynolds number, $R e_{\mathrm{c}}$ & $7.246 \times 10^{4} \times \omega_{\mathrm{c}}$ \\
\hline Marangoni number, $M a_{1}$ & $9.993 \times 10^{7}$ \\
\hline Marangoni number, $\mathrm{Ma}_{2}$ & $2.973 \times 10^{6}$ \\
\hline Peclet number, $P e$ & 0.01278 \\
\hline \multicolumn{2}{|l|}{ Geometry } \\
\hline Crystal radius, $r_{\mathrm{s}}$ & $0.0375 \mathrm{~m}$ \\
\hline Crucible inner radius, $r_{\mathrm{c}}$ & $0.075 \mathrm{~m}$ \\
\hline Melt height, $h_{\ell}$ & $0.035 \mathrm{~m}$ \\
\hline Encapsulant thickness, $h_{\mathrm{e}}$ & $0.004-0.02 \mathrm{~m}$ \\
\hline Crystal height, $h_{\mathrm{s}}$ & \\
\hline
\end{tabular}




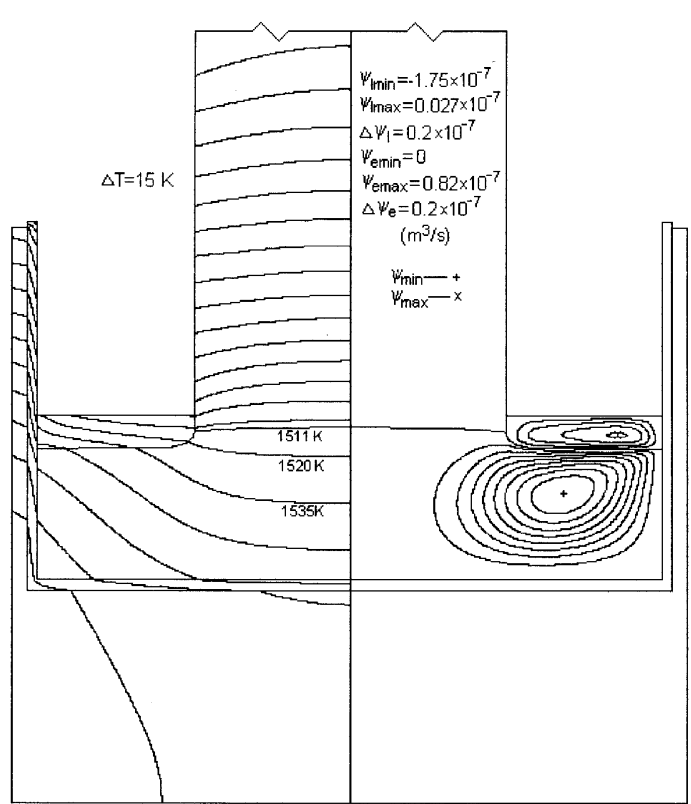

(a)

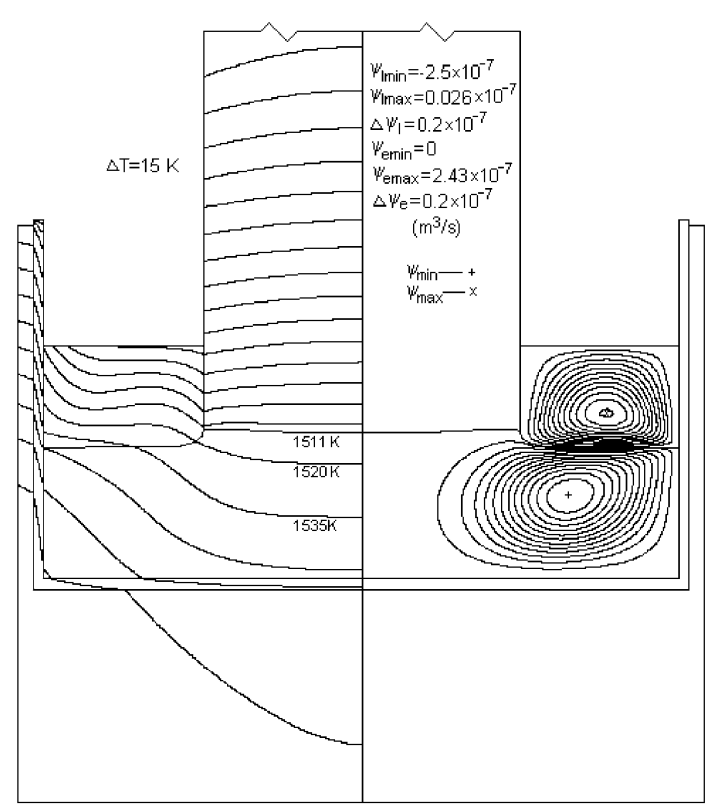

(b)

Fig. 2. Isotherms and contours of stream function ( $M a$-driven flow, $H a=0, \omega_{\mathrm{s}}=\omega_{\mathrm{c}}=0 \mathrm{rpm}$ ): (a) $h_{\mathrm{e}}=0.4 \mathrm{~cm}$; (b) $h_{\mathrm{e}}=2.0 \mathrm{~cm}$.

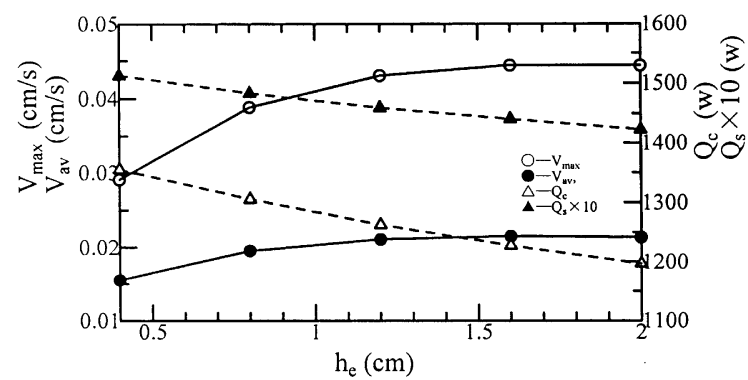

Fig. 3. Maximum velocity $V_{\max }$, average velocity $V_{\mathrm{av}}$, total heat input $Q_{\mathrm{c}}$ and total heat flow $Q_{\mathrm{s}}$ versus the encapsulant thickness $h_{\mathrm{e}}\left(M a\right.$-driven flow, $\left.\mathrm{Ha}=0, \omega_{\mathrm{s}}=\omega_{\mathrm{c}}=0 \mathrm{rpm}\right)$.

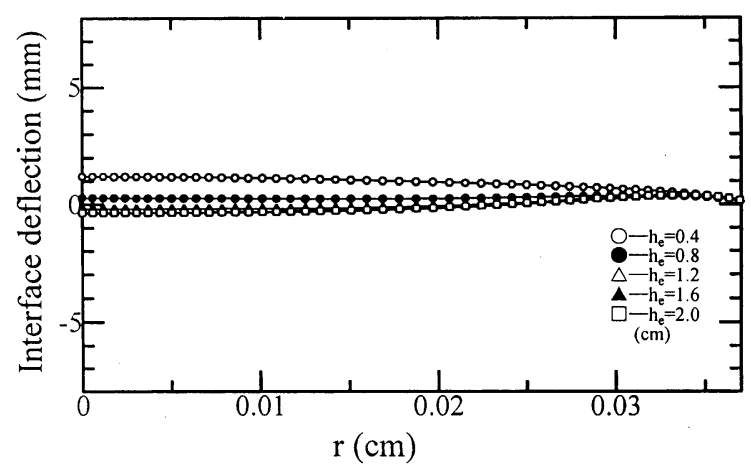

Fig. 4. Interfacial deflection under different $h_{\mathrm{e}}$ ( $M a$-driven flow, $\left.H a=0, \omega_{\mathrm{s}}=\omega_{\mathrm{c}}=0 \mathrm{rpm}\right)$. total heat flow from the melt/crystal interface, $Q_{\mathrm{s}}$, decreases from 135.6 to $119.7 \mathrm{~W}$, as shown in Fig. 3. As a consequence, the concave interface converts into convex except for the part near the periphery as shown in Fig. 4.

(2) Marangoni-driven flow + buoyancy-driven flow. In the case of Marangoni-driven flow combined with buoyancy-driven flow (hereafter we denote it as $M a+B$ driven flow), the flow in the melt is much stronger thereby. The hot fluid is easy to be carried to the internal region so that the interfacial deflection gets very remarkable. The enhanced convective heat transfer distorts the isotherm (see Figs. 5 and 6). Compared with the case of $M a$-driven flow, $Q_{\mathrm{c}}$ is smaller $\left(Q_{\mathrm{c}}\right.$ is in the range $1373-1409 \mathrm{~W})$ and $Q_{\mathrm{s}}$ is larger ( $Q_{\mathrm{s}}$ in the range 150-155 W) as indicated in Fig. 6. The effect of the encapsulant thickness, $h_{\mathrm{e}}$, on the interface shape is obvious but not monotonous as shown in Fig. 7. The maximum deflection of the interface of $4.345 \mathrm{~mm}$ occurs $h_{\mathrm{e}}=2.0 \mathrm{~cm}$, which is 3.6 times larger than that in the case of $M a$ driven flow.

\subsection{Magnetic field strength}

The effect of the magnetic field strength on the flow and heat transfer for a fixed thickness of encapsulant $\left(h_{\mathrm{e}}=1.6 \mathrm{~cm}\right)$, crystal rotation rate $\left(\omega_{\mathrm{s}}=6 \mathrm{rpm}\right)$ and crucible rotation rate $\left(\omega_{\mathrm{s}}=16 \mathrm{rpm}\right)$ in the case of $M a+B$-driven flow is shown in Figs. 8-10. The Ekman layer induced by crucible rotation is observed at the 


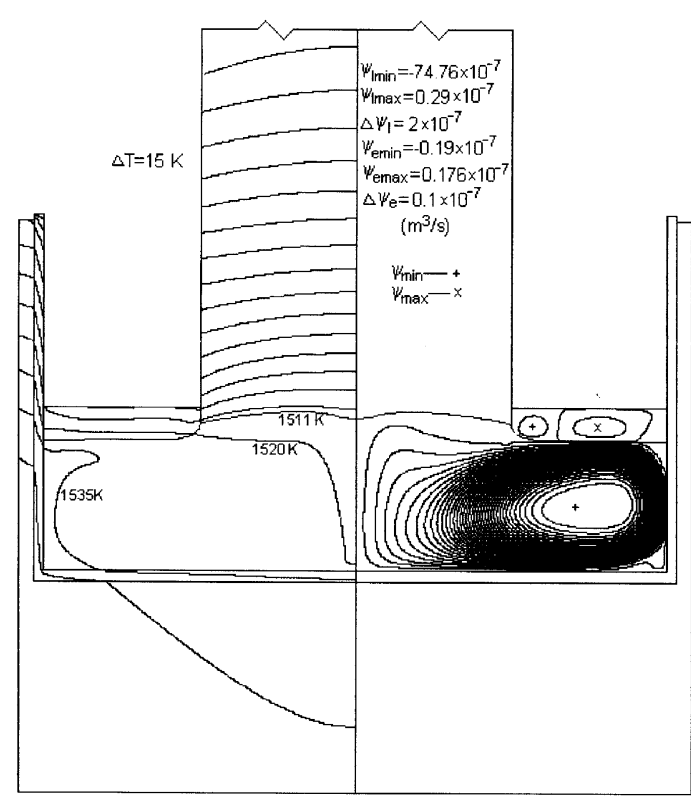

(a)

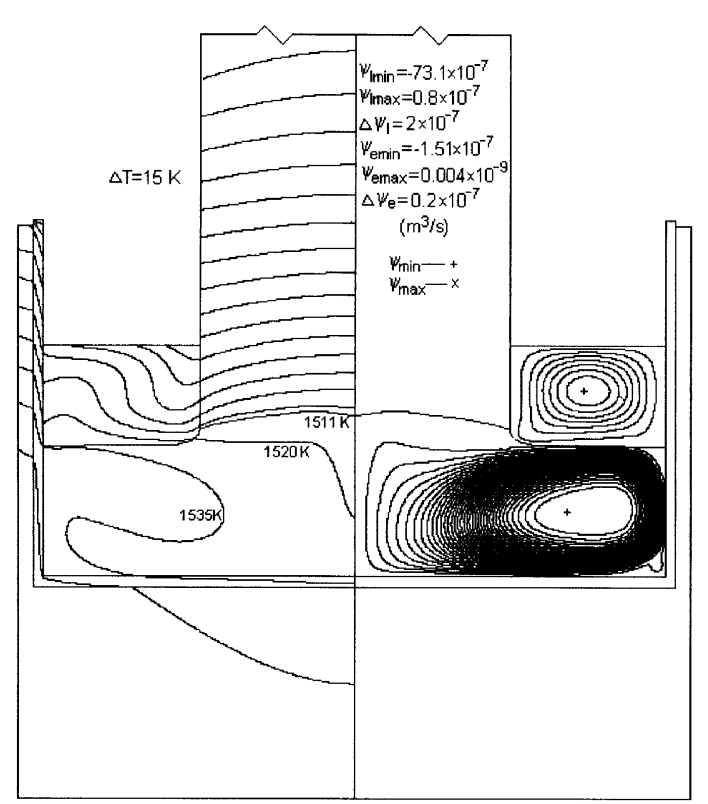

(b)

Fig. 5. Isotherms and contours of stream function $\left(M a+B\right.$-driven flow, $\left.H a=0, \omega_{\mathrm{s}}=\omega_{\mathrm{c}}=0 \mathrm{rpm}\right)$ : (a) $h_{\mathrm{e}}=0.4 \mathrm{~cm}$; (b) $h_{\mathrm{e}}=2.0 \mathrm{~cm}$.

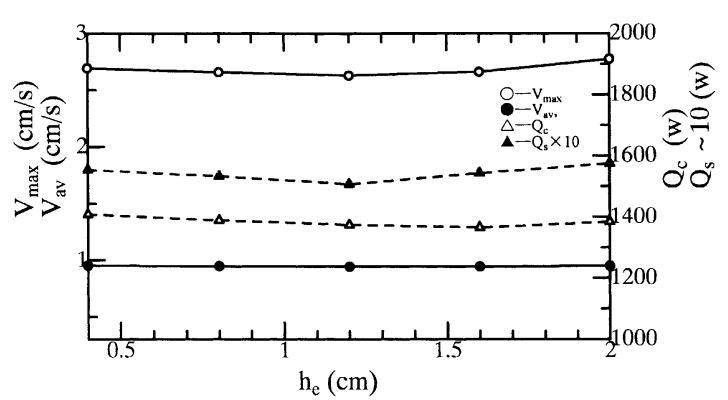

Fig. 6. Maximum velocity $V_{\max }$, average velocity $V_{\mathrm{av}}$, total heat input $Q_{\mathrm{c}}$ and total heat flow $Q_{\mathrm{s}}$ versus the encapsulant thickness $h_{\mathrm{e}}\left(M a+B\right.$-driven flow, $\left.H a=0, \omega_{\mathrm{s}}=\omega_{\mathrm{c}}=0 \mathrm{rpm}\right)$.

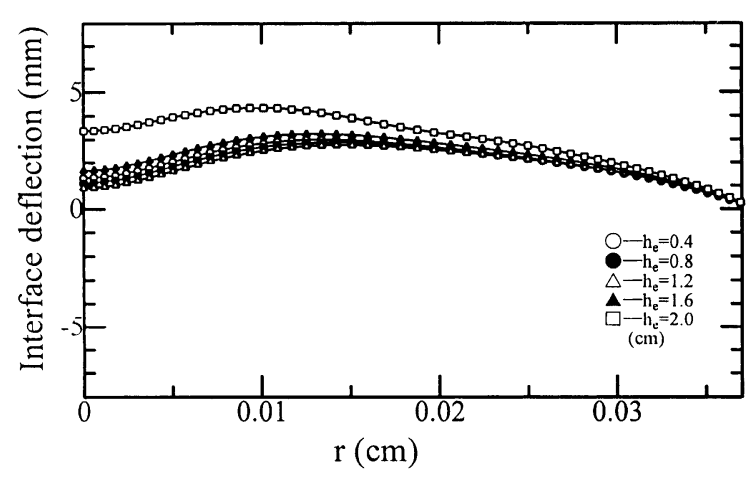

Fig. 7. Interfacial deflection under different thicknesses of the encapsulant ( $M a+B$-driven flow, $\left.H a=0, \omega_{\mathrm{s}}=\omega_{\mathrm{c}}=0 \mathrm{rpm}\right)$. bottom of the crucible and the flow in the melt is reduced effectively with the introduction of an axial magnetic field. When increasing the dimensionless Hartmann number from 120 to 180 , the maximum velocity $V_{\max }$ and the average velocity $V_{\mathrm{av}}$ in the melt decrease by $38 \%$ and $46.8 \%$, respectively, i.e. from $V_{\max }=1.355 \mathrm{~cm} / \mathrm{s}$ and $V_{\mathrm{av}}=0.451 \mathrm{~cm} / \mathrm{s}$ at $H a=120$ to $V_{\max }=0.84 \mathrm{~cm} / \mathrm{s}$ and $V_{\mathrm{av}}=0.24 \mathrm{~cm} / \mathrm{s}$ at $H a=180$, respectively, as indicated in Fig. 9; meanwhile $Q_{\mathrm{c}}$ increases from 1351 to $1363 \mathrm{~W}$ and $Q_{\text {s }}$ decreases from 150.1 to $137.7 \mathrm{~W}$, respectively. The magnetic field has a non-negligible influence on the $M$ type interface. The deflection of the interface increases significantly with the increasing magnetic field strength (see Fig. 10).

\subsection{Crystal and crucible rotations}

Crystal rotation has a remarkable effect on the flow pattern and heat transfer. A new cell appears beneath the melt/crystal interface nearby the periphery and it will get larger with increasing rotation rate of crystal. Particularly, one can see from Fig. 11 that the slender meridional flow cell filling the bulk breaks into two cells for a crystal rotation of $\omega_{\mathrm{s}}=20 \mathrm{rpm}$. The change of the maximum velocity $V_{\max }$ and the average velocity $V_{\mathrm{av}}$ in the melt with crystal rotation rate $\omega_{\mathrm{s}}$ is depicted in Fig. 12. An increase of $38 \%$ for $V_{\max }$ and $17.4 \%$ for $V_{\mathrm{av}}$ is observed when changing the rotation rate of crystal $\omega_{\mathrm{s}}$ from 2 to $20 \mathrm{rpm}$. Due to the more effective heat transfer resulting from the forced convective cell driven by 


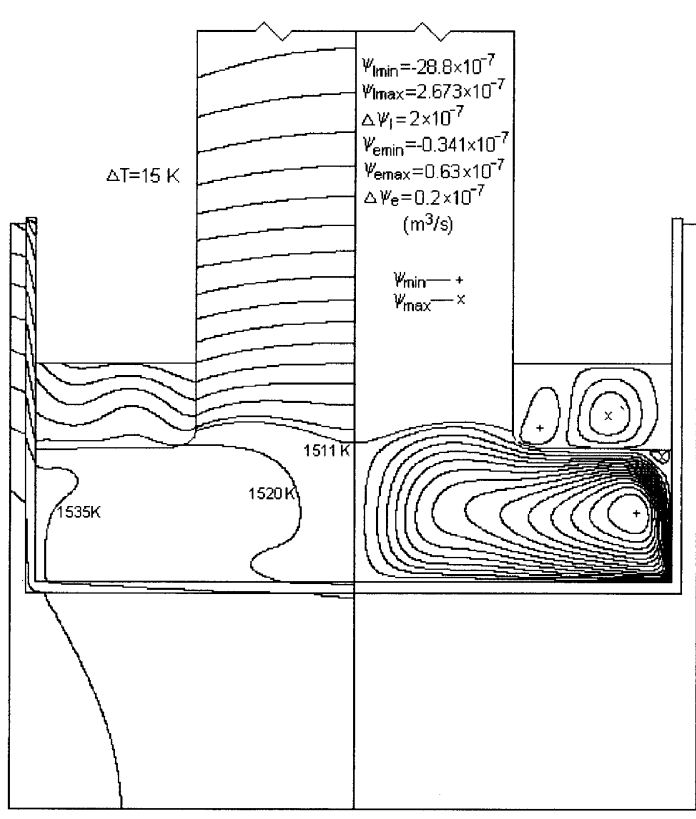

(a)

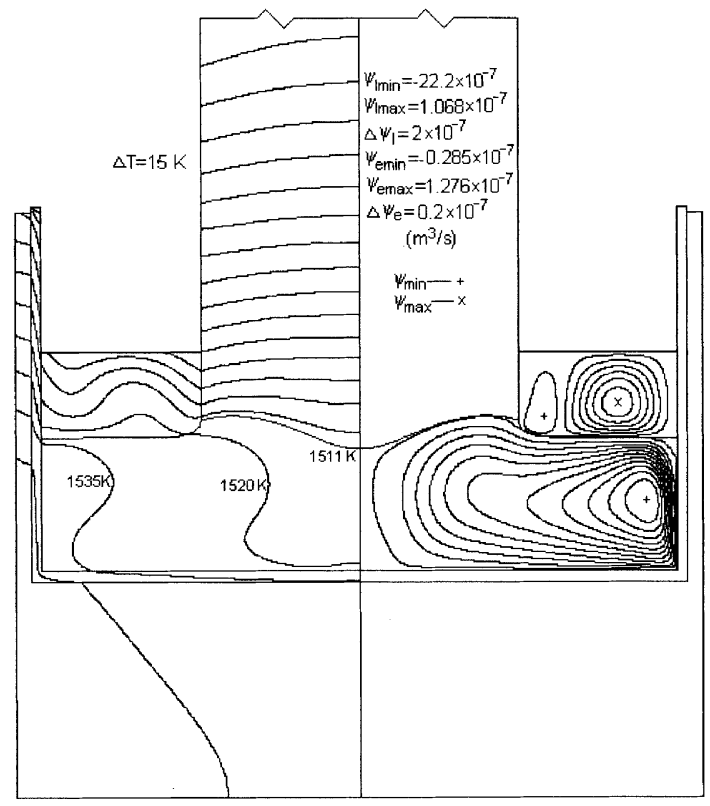

(b)

Fig. 8. Isotherms and contours of stream function $\left(M a+B\right.$-driven flow, $H a=120, \omega_{\mathrm{s}}=6 \mathrm{rpm}$ and $\left.\omega_{\mathrm{c}}=16 \mathrm{rpm}\right)$ : (a) $h_{\mathrm{e}}=1.6 \mathrm{~cm}$, $H a=120$; (b) $h_{\mathrm{e}}=2.0 \mathrm{~cm}, H a=180$.

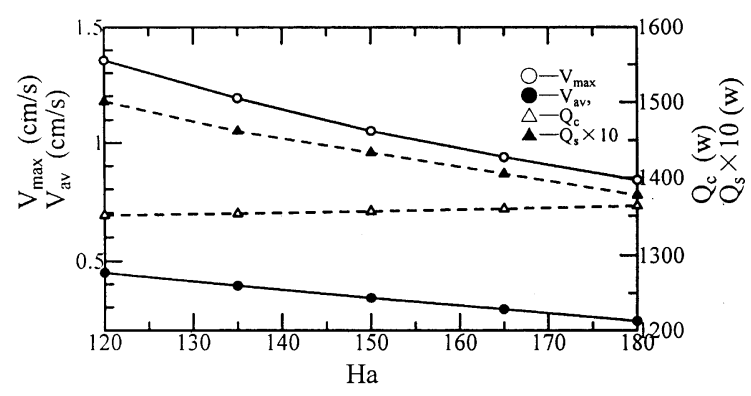

Fig. 9. Maximum velocity $V_{\max }$, average velocity $V_{\mathrm{av}}$, total heat input $Q_{\mathrm{c}}$ and total heat flow $Q_{\mathrm{s}}$ versus Hartmann number $\mathrm{Ha}$ $\left(M a+B\right.$-driven flow, $\left.h_{\mathrm{e}}=1.6 \mathrm{~cm}, \omega_{\mathrm{s}}=6 \mathrm{rpm}, \omega_{\mathrm{c}}=16 \mathrm{rpm}\right)$.

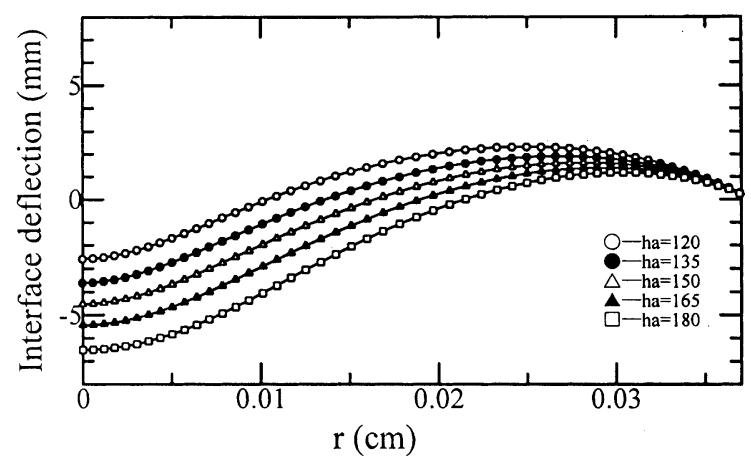

Fig. 10. Interfacial deflection under different Hartmann numbers $\left(M a+B\right.$-driven flow, $\left.h_{\mathrm{e}}=1.6, \omega_{\mathrm{s}}=6 \mathrm{rpm}, \omega_{\mathrm{c}}=16 \mathrm{rpm}\right)$. crystal rotation, the total heat input $Q_{\mathrm{c}}$ and total heat flux from melt/crystal interface $Q_{\text {s }}$ get large (see Fig. 12). Consequently the interface of $M$-type trends to concave, and thus, to carry away more heat from the melt/crystal interface (see Fig. 13).

On the contrary, the effect of the crucible rotation on the flow and heat transfer is not so obvious, as shown in Fig. 14. As indicated in reference [14], in the case of adopting axial magnetic field, crystal rotation appears to be the more important parameter.

\section{Conclusions}

A global simulation code was applied to numerically study the characteristics of flow and heat transfer in the LEC growth of CaAs with and without an axial magnetic field. The results indicate that:

1. Compared with Marangoni combined buoyancydriven flow, the pure Marangoni-driven flow is so weak that the heat transfer is thermal conductiondominated.

2. By increasing the thickness of encapsulant, the crystal, which is initially concave at the interface, becomes convex except for the part near the periphery in the case of pure Marangoni-driven flow. The thickness of encapsulant has a more remarkable influence on the interface shape in the case of Marangoni combined 


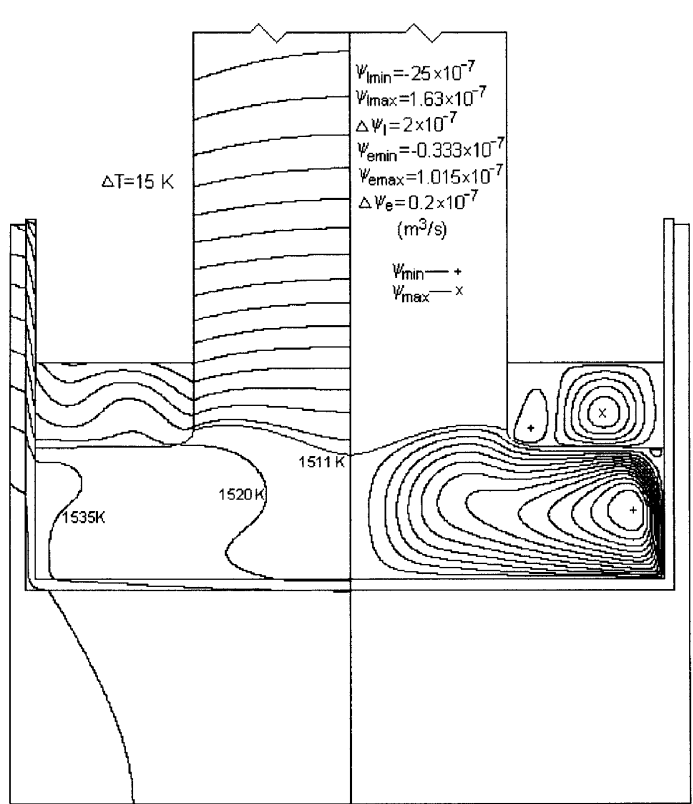

(a)

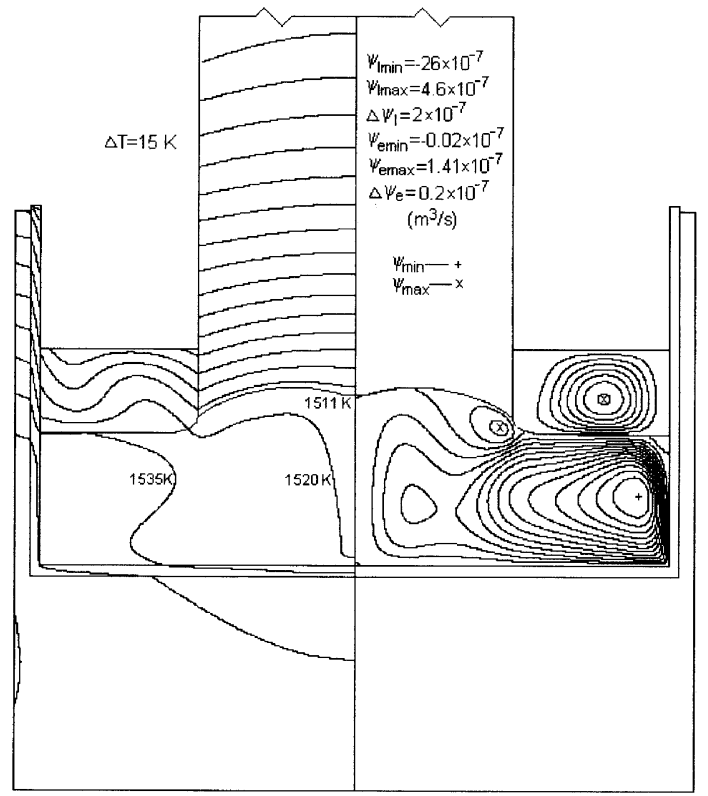

(b)

Fig. 11. Isotherms and contours of stream function $\left(M a+B\right.$-driven flow, $\left.h_{\mathrm{e}}=1.6 \mathrm{~cm}, H a=150, \omega_{\mathrm{c}}=16 \mathrm{rpm}\right):(\mathrm{a}) h_{\mathrm{e}}=1.6 \mathrm{~cm}$, $\omega_{\mathrm{s}}=2 \mathrm{rpm} ;$ (b) $h_{\mathrm{e}}=2.0 \mathrm{~cm}, \omega_{\mathrm{s}}=20 \mathrm{rpm}$.

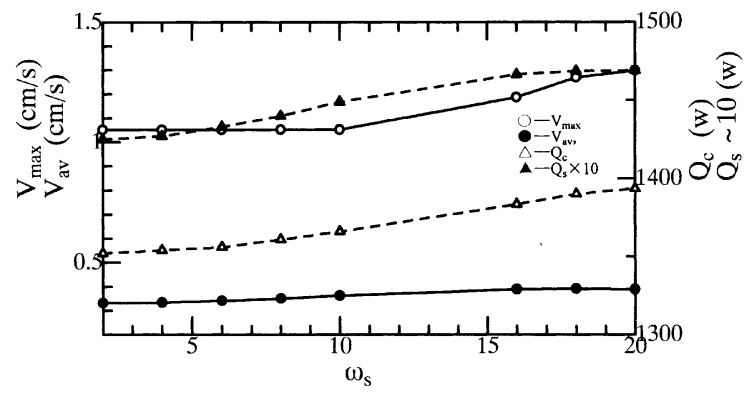

Fig. 12. Maximum velocity $V_{\max }$, average velocity $V_{\mathrm{av}}$, total heat input $Q_{\mathrm{c}}$ and total heat flow $Q_{\mathrm{s}}$ versus crystal rotation rate $\omega_{\mathrm{s}}$ $\left(M a+B\right.$-driven flow with $\left.h_{\mathrm{e}}=1.6 \mathrm{~cm}, \mathrm{Ha}=150, \omega_{\mathrm{c}}=16 \mathrm{rpm}\right)$.

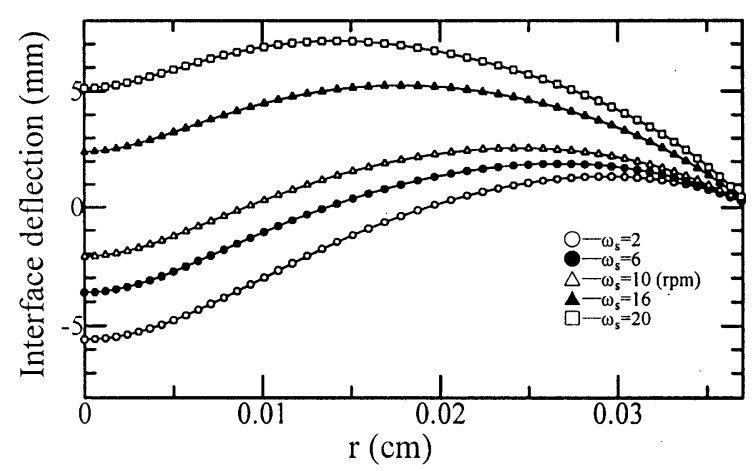

Fig. 13. Interfacial deflection under different crystal rotation rates $\omega_{\mathrm{s}}\left(M a+B\right.$-driven flow, $\left.h_{\mathrm{e}}=1.6 \mathrm{~cm}, H a=150, \omega_{\mathrm{c}}=16 \mathrm{rpm}\right)$.

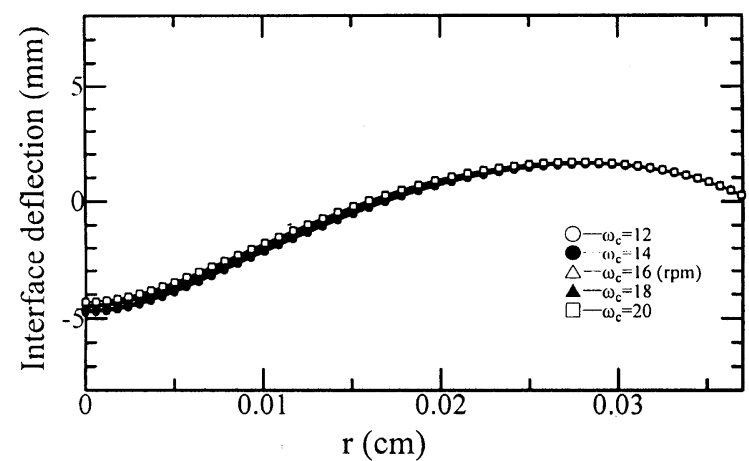

Fig. 14. Interfacial deflection under different crucible rotation rates $\omega_{\mathrm{c}}\left(M a+B\right.$-driven flow with $h_{\mathrm{e}}=1.6 \mathrm{~cm}, H a=150$, $\left.\omega_{\mathrm{s}}=6 \mathrm{rpm}\right)$.

with buoyancy driven flow. The maximum interfacial deflection can reach $4.345 \mathrm{~mm}$.

3. By imposing an axial magnetic field, the convection in the melt is reduced effectively. With increasing magnetic field strength, the interfacial deflection increases significantly.

4. The rotation of the crystal has a remarkable effect on the flow and heat transfer, multi-cell flow pattern may appear in the melt. With increasing crystal rotation rate the $M$-type interface trends to concave as a whole. On the contrary, the effect of the crucible rotation on the flow and the heat transfer is not so obvious. 
5. The total heat input from the crucible and total heat flux from the melt/crystal interface depend on the thickness of the encapsulant, magnetic field strength and crystal rotation rate.

\section{Acknowledgements}

This work was supported by National Microgravity Laboratory, Institute of Mechanics Chinese Academy of Sciences.

\section{References}

[1] Mingwei Li, Yourong Li, Nobuyuki Imaishi, Taka Tsukada, Global simulation of a silicon Czochralski furnace, J. Cryst. Growth 234 (1) 32-46.

[2] F. Dupret, P. Nicodeme, Y. Ryckmans, P. Wouters, M.J. Crochet, Numerical method for reducing stress level in GaAs crystal, J. Cryst. Growth 97 (1989) 162-172.

[3] F. Dupret, P. Nicodeme, Y. Ryckmans, P. Wouters, M.J. Crochet, Global modeling of heat transfer in crystal growth furnaces, Int. J. Heat Mass Transfer 33 (9) (1990) 1849-1871.

[4] P.D. Thomas, J.J. Derby, L.J. Atherton, R.A. Brown, Dynamics of liquid-encapsulanted Czochralski growth of gallium arsenide: comparing model with experiment, J. Cryst. Growth 96 (1989) 135-152.
[5] J. Fainberg, H.-J. Leister, G. Muller, Numerical simulation of the LEC-growth of GaAs crystals with account of highpressure gas convection, J. Cryst. Growth 180 (1997) 517523.

[6] V.I. Polezhaev, O.A. Bessonov, N.V. Nikitin, S.A. Nikitin, Convective interaction and instabilities in GaAs Czochralski model, J. Cryst. Growth 230 (2001) 40-47.

[7] U. Rehse, W. Miller, Ch. Frank, P. Rudolph, M. Neubert, A numerical investigation of the effects of iso- and counterrotation on the shape of the $\mathrm{VCz}$ growth interface, J. Cryst. Growth 230 (2001) 143-147.

[8] X. Geng, X.B. Wu, Z.Y. Guo, Numerical simulation of combined flow in Czochralski crystal growth, J. Cryst. Growth 179 (1997) 309-319.

[9] M.E. Salcudean, P. Sabhapathy, F. Weinberg, Numerical study of free and forced convection in the LEC growth of GaAs, J. Cryst. Growth 94 (1989) 522-526.

[10] P. Sabhapathy, M.E. Salcubean, Numerical study of flow and heat transfer in LEC growth of GaAs with an axial magnetic field, J. Cryst. Growth 104 (1990) 371-388.

[11] Q.S. Liu, B. Roux, M.G. Velarde, Thermocapillary convection in two-layer systems, Int. J. Heat Mass Transfer 41 (11) (1998) 1499-1511.

[12] J. Li, J. Sun, Z. Saghir, Buoyant and thermocapillary flow in liquid encapsulated float zone, J. Cryst. Growth 131 (1993) 83-96.

[13] Institute of Thermophysical property of Japan, in: Thermophysical Properties Handbook, 1990, p. 241 (in Japanese).

[14] D.T.J. Hurle, R.W. Series, in: Handbook of Crystal Growth, 1994, p. 267. 